\title{
Testing Weak Form Market Efficiency and its Impact on Stock Valuation: Empirical Evidence from Stock Market in Bangladesh
}

\author{
Riyashad Ahmed ${ }^{1}$, Md Delowar Hossain ${ }^{2}$ \\ ${ }^{1,2}$ BRAC Business School, BRAC University, Bangladesh \\ E-mail: delowar.hossain@teachforbangladesh.org
}

Received: December 28, 2018; Accepted: February 12, 2019; Published: March 30, 2019

Permalink/DOI: http://dx.doi.org/10.17977/um002v11i12019p001

\begin{abstract}
This empirical study focuses on the stock valuation considering weak form market efficiency. The objective is to find out whether the stock market in Bangladesh is efficient or not by testing the weak form market efficiency. Here, daily stock trading prices from the Dhaka Stock Exchange (DSE) were considered during the period from 2011 till 2017. A total of 26 leading companies were randomly selected and their intrinsic stock prices were calculated. CAPM model is used to find out the intrinsic value. It can be concluded that if the market price and the intrinsic value of a firm are equal, then the market is believed to be weak form efficient as only historical information is reflected on the stock prices. Our result shows that Dhaka Stock Exchange is not a weak form efficient market as the market prices and intrinsic values are not equal for the considered corporations. Arbitrage profit may be possible to generate by our investors.
\end{abstract}

Keywords: Market Efficiency; Weak Form efficiency; Intrinsic Value; CAPM; Undervalued and Overvalued.

JEL Classification: G10, G11, G14

\section{INTRODUCTION}

When a capital market is efficient, technical analysts generally fail to gain an arbitrage profit. However, they keep analyzing the market constantly so that they might achieve that abnormal profit. If a market does not follow the random walk and is not efficient either, they achieve their desired profit. Malkiel and Fama (1970) developed the theory of efficient capital markets and suggested three forms of capital market efficiency; namely - weak form efficiency, semi-strong form efficiency, and strong form efficiency. Informed and knowledgeable investors tend to apply these techniques to assess the market. In a weak form market efficiency, only historical information is reflected in the share price. When both historical information and publicly available information are reflected in the stock price, it is said to be the semi-strong form market efficiency and in this scenario, the technical analysts cannot achieve the abnormal profit. The remaining one is the strong form of market efficiency, where both publicly and privately available information is reflected in the stock price and no one can achieve abnormal profit. In this study, the Capital Asset Pricing Model (CAPM) is used to find out the intrinsic value of a stock. Intrinsic value is the fair market price that is used to evaluate a particular stock whether it is overvalued or undervalued. If the market 
price is less than the intrinsic value, it is undervalued. If the market price is more than the intrinsic value, it is considered to be an overvalued stock. Now the question is, why this model is called the pricing model and how is it presented? We have used this pricing model to find out the intrinsic value that helps us to understand whether a stock price is fair or not.

The CAPM model is represented by the securities market line (SML). SML is a straight line that represents all the assets that are traded in the market. Each point of SML represents the relationship between the expected rerun and beta. Here beta is the systematic risk which represents an asset's responsiveness to a changing market condition. Here, we would like to address how the SML helps to find the intrinsic value of an asset. If an asset's beta is known, it is possible to calculate the required rate of return by using the CAPM model and hence we can come up with the intrinsic value by using an appropriate stock valuation technique. If the expected return is higher than the present return, the market is considered to be undervalued. Therefore, the stock price is expected to increase in the near future. Again, a higher return is generally associated with higher risk. Since the risk premium is increased due to undertaking higher risk in the capital market, the reward generally increases as well. There are two types of risks an investor has to bear: firm-specific risk and systematic risk. A firm specific risk may be reduced and eliminated by proper diversification and the rational investors are always advised to follow that. On the other hand, the systematic risk (market risk) is the relevant risk for a firm, hence the companies are more concerned about it. In this study, systematic risks of various sample corporations were embedded in the CAPM model to come up to our conclusion.

\section{LITERATURE REVIEW}

When an investor wants to invest in achieving arbitrage profit from the stock market, it is necessary to know the market efficiency. At present different technical analyst analyze the market to know the market efficiency. When an investor wants to invest in a capital market they obviously consider the required rate of return because they want to know the minimum return without which investors do not want to invest. It is necessary to identify the market is efficiency or not. The study on market efficiency finds out that Bangladesh along with other fifteen Asian countries is considered as inefficiency. Here, investors can earn arbitrage profit. Different studies on CAPM return conducted by Jagannathan and Wang (1996), and Lewellen and Nagel (2006) where they focused on condition CAPM and the expected return. Bakshi and Chen (2005) studied on stock valuation in dynamic economic. They consider three inputs to evaluate the stock which are net earnings per share, expected earnings growth, and interest rate. Pástor and Pietro (2003) conducted a study on stock valuation and learning about profitability with considering the market to book ratio MB. This research gives the evidence that the younger stock which pays no dividend provides a more volatile return.

Srinivasan (2010) examined the weak form efficiency of Indian stoic market based on daily observation from 1997 to 2010 by using unit root test. The result of this study finds that the market is not efficient. It has the opportunity to achieve the arbitrage profit. By making diversified portfolio investors can achieve profit.

Gupta and Basu (2007) examined the weak form efficiency based on the random walk hypothesis on two equity markets in India by considering the period 
from 1991 to 2006. It suggests that the markets do not follow the random walk model and it rejects the weak form efficiency hypothesis.

Mobarek et al. (2008) studied on market efficiency on Bangladesh stock market using the daily index of Dhaka stock exchange based on historical data from 1988 to 2000 by applying both parametric test and non-parametric test. The finding of this study is that the market does not follow the random walk. Therefore the technical analyst can achieve arbitrage profit from the market.

Poshakwale (1996) tested the Indian stock market with the title evidenced on weak form efficiency and day of the week effect in the Indian stock market considering data from 1987 to 1994 . The study gives the result that the market is not efficient and random walk theory does not apply on this market.

In our research, we extended our previous research which is on Testing Weak form Market Efficiency: Empirical Evidence from Selected Asian Stock Markets by Ahmed and Hossain (2018) using a different module to know the market efficiency. In the $2^{\text {nd }}$ section, we give the methodology about the module, $3^{\text {rd }}$ section is the analysis part and after that conclusion and in last point reference is concluded.

\section{METHOD}

\section{Data}

The historical stock price information is considered from the period 2011 to 2017. The following 26 leading companies from the capital market were considered: Aamra Technologies Limited; Ab Bank; Aci Formulations Limited; Active Fine Chemicals Ltd; Active Fine Chemicals Ltd; Alhaj Textile Mills Limited; Apex Foods Limited; Anwar Galvanizing Limited; Aims First Guaranteed Mutual Fund; Agrani Insurance Co. Ltd.; Aftab Automobiles Limited; Bangas Limited; Bay Leasing \& Investment Limited; Bsrm Steels Limited; Desh Garments Limited; Golden Son Ltd.; Jute Spinners Limited; Intech Online Limited.; Idlc Finance Limited; H.R. Textile Mills Limited; Gph Ispat Ltd.; Keya Cosmetics Ltd.; Lafarge Surma Cement Ltd.; Lankabangla Finance Ltd.; Libra Infusions Ltd.; Marico Bangladesh Limited; Metro Spinning Ltd.; Mjl Bangladesh Limited; Navana Cng Limited; Orion Infusion Ltd.; and United Airways (BD) Ltd. These 26 corporations were selected from different industries by using random sampling method to get an overall idea about the entire stock market. The corporations mentioned above were selected from the following industries: banking, financial institutions, textile, food and beverage, cement, ceramic, fuel and power, and miscellaneous. Daily index data from Dhaka stock exchange broad index is considered for calculating the return. The equation for calculating the rate of return is as follows:

$$
\text { Return }=\ln \frac{P t}{P t-1}
$$

Here,

$\mathrm{Pt}=$ market price of a stock at the time of " $\mathrm{t}$ "

$\mathrm{Pt}-1=$ market price of a stock at the time of " $\mathrm{t}-1$ "

\section{CAPM Formulation}

The most important part of this research is the model that we have used to find out the fair value of the stocks. Numerous analysts introduced the CAPM 
model in their studies. For example, Jack Treynor (1961, 1962), William F. Sharpe (1964), John Lintner (1965) and Jan Mossin (1966) used the CAPM model in their valuation studies.

The following formula was used to find out the required rate of return:

$$
\mathrm{E}(\mathrm{Ri})=\mathrm{Rf}+(\mathrm{Rm}-\mathrm{RF}) \beta
$$

The Stock market return was found by:

$R m=\frac{\text { Ending of Month Stock Price }}{\text { Beginning of Month Stock Price }}-1$

The market risk $(\beta)$ was calculated by:

$\beta=$ slope of the best-fit line in scatter diagram

One year maturity Treasury bill (T-bill) issued by the Bangladesh Government yields $7.5 \%$ per annum and was considered as the risk-free rate of return for this study.

\section{Intrinsic Value}

$\mathrm{P}=\mathrm{D} / \mathrm{E}(\mathrm{Ri})$

$\mathrm{D}=$ Current Price $\mathrm{X}$ Market Return

The intrinsic value is considered as the fair market price and this price helps us to assess the market efficiency. In an efficient market condition, the intrinsic value and current trading price are expected to be equal.

\section{RESULTS AND DISCUSSION \\ Descriptive Statistics}

In the table- 1 and table - 2 below, descriptive statistics of the considering companies are disclosed. Among the selected corporations, returns from nine companies tend to be negatively skewed which gives an indication that the empirical returns for these corporations seem to be more negative than positive. However, other remaining company's returns were positively skewed. The average daily return was highest for stock 10 , which was 0.002 with a standard deviation of 0.034 . On the other hand, the stock 13 yielded the maximum negative return (0.003 ) with a standard deviation of .09 .

Table 1. Descriptive Statistics from $1^{\text {st }}$ to $13^{\text {th }}$ Companies

\begin{tabular}{|c|c|c|c|c|c|c|c|c|c|c|c|c|c|}
\hline Details & $\begin{array}{c}\text { Stoc } \\
\text { k } 1\end{array}$ & $\begin{array}{c}\text { Stoc } \\
\text { k } 2\end{array}$ & $\begin{array}{c}\text { Stoc } \\
\text { k } 3\end{array}$ & $\begin{array}{c}\text { Stoc } \\
\text { k } 4\end{array}$ & $\begin{array}{c}\text { Stoc } \\
\text { k } 5\end{array}$ & $\begin{array}{c}\text { Stoc } \\
\text { k } 6\end{array}$ & $\begin{array}{c}\text { Stoc } \\
\text { k } 7\end{array}$ & $\begin{array}{c}\text { Stock } \\
8\end{array}$ & $\begin{array}{c}\text { Stock } \\
9\end{array}$ & $\begin{array}{c}\text { Stock } \\
10\end{array}$ & $\begin{array}{c}\text { Stock } \\
11\end{array}$ & $\begin{array}{c}\text { Stock } \\
12\end{array}$ & $\begin{array}{l}\text { Stoc } \\
\text { k } 13\end{array}$ \\
\hline Mean & 0 & 0 & $\begin{array}{r}0.00 \\
1\end{array}$ & $\begin{array}{r}- \\
0.00 \\
1\end{array}$ & 0 & $\begin{array}{r}0.00 \\
1\end{array}$ & 0 & 0 & 0 & 0.002 & 0.001 & 0.001 & $\begin{array}{r}- \\
0.00 \\
3\end{array}$ \\
\hline $\begin{array}{l}\text { Standar } \\
\mathrm{d} \\
\text { Error }\end{array}$ & $\begin{array}{r}0.00 \\
1\end{array}$ & $\begin{array}{r}0.00 \\
1\end{array}$ & $\begin{array}{r}0.00 \\
1\end{array}$ & $\begin{array}{r}0.00 \\
1\end{array}$ & $\begin{array}{r}0.00 \\
1\end{array}$ & $\begin{array}{r}0.00 \\
1\end{array}$ & $\begin{array}{r}0.00 \\
1\end{array}$ & 0.001 & 0.001 & 0.001 & 0 & 0 & $\begin{array}{r}0.00 \\
3\end{array}$ \\
\hline
\end{tabular}




\begin{tabular}{|c|c|c|c|c|c|c|c|c|c|c|c|c|c|}
\hline Median & $\begin{array}{r}- \\
0.00 \\
3\end{array}$ & 0 & $\begin{array}{r}- \\
0.00 \\
2\end{array}$ & $\begin{array}{r}- \\
0.00 \\
2\end{array}$ & 0 & $\begin{array}{r}- \\
0.00 \\
3\end{array}$ & $\begin{array}{r}- \\
0.00 \\
3\end{array}$ & 0 & $0.002^{-}$ & 0 & 0 & 0 & $\begin{array}{r}- \\
0.00 \\
3\end{array}$ \\
\hline Mode & 0 & 0 & 0 & 0 & 0 & 0 & 0 & 0 & 0 & 0 & 0 & 0 & 0 \\
\hline $\begin{array}{l}\text { Standar } \\
\text { d } \\
\text { Deviati } \\
\text { on }\end{array}$ & $\begin{array}{r}0.02 \\
6\end{array}$ & $\begin{array}{r}0.02 \\
2\end{array}$ & $\begin{array}{r}0.02 \\
4\end{array}$ & $\begin{array}{r}0.02 \\
2\end{array}$ & $\begin{array}{r}0.02 \\
1\end{array}$ & 0.03 & $\begin{array}{r}0.02 \\
8\end{array}$ & 0.026 & 0.021 & 0.034 & 0.014 & 0.016 & 0.09 \\
\hline $\begin{array}{l}\text { Sample } \\
\text { Varianc } \\
\text { e }\end{array}$ & $\begin{array}{r}0.00 \\
1\end{array}$ & 0 & $\begin{array}{r}0.00 \\
1\end{array}$ & 0 & 0 & $\begin{array}{r}0.00 \\
1\end{array}$ & $\begin{array}{r}0.00 \\
1\end{array}$ & 0.001 & 0 & 0.001 & 0 & 0 & $\begin{array}{r}0.00 \\
8\end{array}$ \\
\hline Kurtosis & 2.3 & 5.5 & 3.4 & 36.6 & 21.8 & 2 & 1.7 & 2.3 & 3.7 & 2 & 5.3 & 2.7 & $\begin{array}{r}1024 \\
.6\end{array}$ \\
\hline $\begin{array}{l}\text { Skewne } \\
\text { ss }\end{array}$ & 0.5 & 0.1 & 0.8 & -3 & -1.1 & 0.6 & 0.7 & 0.1 & 0.9 & 0.1 & 0.9 & 0.5 & 30.8 \\
\hline Range & 0.2 & 0.3 & 0.2 & 0.4 & 0.4 & 0.2 & 0.2 & 0.2 & 0.2 & 0.3 & 0.1 & 0.1 & 3.1 \\
\hline $\begin{array}{l}\text { Minimu } \\
\mathrm{m}\end{array}$ & -0.1 & -0.2 & -0.1 & -0.3 & -0.2 & -0.1 & -0.1 & -0.1 & -0.1 & -0.2 & -0.1 & -0.1 & -3 \\
\hline $\begin{array}{l}\text { Maximu } \\
\mathrm{m}\end{array}$ & $\begin{array}{r}0.09 \\
5\end{array}$ & $\begin{array}{r}0.09 \\
4\end{array}$ & $\begin{array}{r}0.09 \\
6\end{array}$ & $\begin{array}{r}0.09 \\
5\end{array}$ & $\begin{array}{r}0.16 \\
3\end{array}$ & $\begin{array}{r}0.09 \\
5\end{array}$ & $\begin{array}{r}0.09 \\
5\end{array}$ & 0.095 & 0.095 & 0.095 & 0.072 & 0.061 & $\begin{array}{r}0.09 \\
5\end{array}$ \\
\hline Sum & $\begin{array}{r}- \\
0.09 \\
2\end{array}$ & $\begin{array}{r}- \\
0.38 \\
9\end{array}$ & $\begin{array}{r}0.86 \\
4\end{array}$ & $\begin{array}{r}- \\
0.68 \\
5\end{array}$ & $\begin{array}{r}- \\
0.04 \\
1\end{array}$ & $\begin{array}{r}1.55 \\
6\end{array}$ & $\begin{array}{r}0.43 \\
9\end{array}$ & -0.36 & -0.29 & 2.136 & 0.806 & 1.401 & $\begin{array}{r}- \\
3.71\end{array}$ \\
\hline Count & 1201 & 1200 & 1201 & 1201 & 1201 & 1201 & 1201 & 1200 & 1201 & 1201 & 1201 & 1201 & 1201 \\
\hline
\end{tabular}

Source: developed by authors; the significance at 5\%.

Table 2. Descriptive Statistics from $14^{\text {th }}$ to $26^{\text {th }}$ Companies

\begin{tabular}{|c|c|c|c|c|c|c|c|c|c|c|c|c|c|}
\hline Details & $\begin{array}{l}\text { Stoc } \\
\text { k } 14\end{array}$ & $\begin{array}{l}\text { Stoc } \\
\text { k } 15\end{array}$ & $\begin{array}{l}\text { Stoc } \\
\text { k } 16\end{array}$ & $\begin{array}{l}\text { Stoc } \\
\text { k } 17\end{array}$ & $\begin{array}{c}\text { Stock } \\
18\end{array}$ & $\begin{array}{l}\text { Stoc } \\
\text { k } 19\end{array}$ & $\begin{array}{l}\text { Stoc } \\
\text { k 20 }\end{array}$ & $\begin{array}{c}\text { Stock } \\
21\end{array}$ & $\begin{array}{c}\text { Stock } \\
22\end{array}$ & $\begin{array}{c}\text { Stock } \\
23\end{array}$ & $\begin{array}{l}\text { Stoc } \\
\text { k } 24\end{array}$ & $\begin{array}{c}\text { Stock } \\
25\end{array}$ & $\begin{array}{c}\text { Stock } \\
26\end{array}$ \\
\hline Mean & 0 & 0 & 0 & 0 & $\begin{array}{r}- \\
0.001\end{array}$ & 0 & 0 & 0.001 & 0 & 0 & $\begin{array}{r}0.00 \\
1\end{array}$ & 0 & 0.001 \\
\hline $\begin{array}{l}\text { Standar } \\
\mathrm{d} \\
\text { Error }\end{array}$ & $\begin{array}{r}0.00 \\
1\end{array}$ & 0 & $\begin{array}{r}0.00 \\
1\end{array}$ & $\begin{array}{r}0.00 \\
1\end{array}$ & 0.001 & $\begin{array}{r}0.00 \\
1\end{array}$ & $\begin{array}{r}0.00 \\
1\end{array}$ & 0.001 & 0.001 & 0.001 & $\begin{array}{r}0.00 \\
1\end{array}$ & 0.001 & 0.001 \\
\hline Median & $\begin{array}{r}- \\
0.00 \\
4\end{array}$ & $\begin{array}{r}- \\
0.00 \\
1\end{array}$ & 0 & 0 & $\begin{array}{r}- \\
0.004\end{array}$ & 0 & $\begin{array}{r}- \\
0.00 \\
2\end{array}$ & 0 & 0 & 0 & $\begin{array}{r}- \\
0.01\end{array}$ & -0 & 0 \\
\hline
\end{tabular}


Ahmed, R. \& Hossain D. M. / Jurnal Ekonomi dan Studi Pembangunan, 11(1), 1 - 13

\begin{tabular}{|c|c|c|c|c|c|c|c|c|c|c|c|c|c|}
\hline $\begin{array}{l}\text { Standar } \\
\text { d } \\
\text { Deviati } \\
\text { on }\end{array}$ & $\begin{array}{r}0.02 \\
9\end{array}$ & $\begin{array}{r}0.01 \\
7\end{array}$ & $\begin{array}{r}0.02 \\
4\end{array}$ & $\begin{array}{r}0.02 \\
9\end{array}$ & 0.024 & 0.02 & $\begin{array}{r}0.01 \\
8\end{array}$ & 0.034 & 0.024 & 0.021 & $\begin{array}{r}0.03 \\
3\end{array}$ & 0.021 & 0.023 \\
\hline $\begin{array}{l}\text { Sample } \\
\text { Varienc } \\
\text { e }\end{array}$ & $\begin{array}{r}0.00 \\
1\end{array}$ & 0 & $\begin{array}{r}0.00 \\
1\end{array}$ & $\begin{array}{r}0.00 \\
1\end{array}$ & 0.001 & 0 & 0 & 0.001 & 0.001 & 0 & $\begin{array}{r}0.00 \\
1\end{array}$ & 0 & 0.001 \\
\hline Kurtosis & 3.4 & 12.4 & 2.6 & 44.7 & 9.2 & 3 & 4.2 & 9.9 & 9.4 & 7.6 & 9.1 & 2.6 & 53.6 \\
\hline $\begin{array}{l}\text { Skewne } \\
\text { ss }\end{array}$ & 0.3 & -0.2 & 0.8 & -2.6 & -0.5 & 0.1 & 0.7 & -0.8 & -0.1 & -0.5 & 1.3 & 0.5 & -3.7 \\
\hline Range & 0.3 & 0.2 & 0.2 & 0.5 & 0.3 & 0.2 & 0.2 & 0.5 & 0.3 & 0.3 & 0.5 & 0.2 & 0.4 \\
\hline $\begin{array}{l}\text { Minimu } \\
\mathrm{m}\end{array}$ & -0.2 & -0.2 & -0.1 & -0.4 & -0.2 & -0.1 & -0.1 & -0.3 & -0.2 & -0.2 & -0.2 & -0.1 & -0.3 \\
\hline $\begin{array}{l}\text { Maximu } \\
\mathrm{m}\end{array}$ & 0.14 & $\begin{array}{r}0.08 \\
5\end{array}$ & $\begin{array}{r}0.09 \\
5\end{array}$ & $\begin{array}{r}0.09 \\
4\end{array}$ & 0.095 & $\begin{array}{r}0.09 \\
5\end{array}$ & $\begin{array}{r}0.09 \\
2\end{array}$ & 0.183 & 0.095 & 0.104 & $\begin{array}{r}0.33 \\
4\end{array}$ & 0.112 & 0.092 \\
\hline Sum & $\begin{array}{r}- \\
0.59 \\
6\end{array}$ & $\begin{array}{r}0.05 \\
6\end{array}$ & $\begin{array}{r}- \\
0.08 \\
2\end{array}$ & $\begin{array}{r}- \\
0.09 \\
6\end{array}$ & $\begin{array}{r}- \\
1.363\end{array}$ & $\begin{array}{r}- \\
0.57\end{array}$ & $\begin{array}{r}0.17 \\
5\end{array}$ & 0.714 & $\begin{array}{r}- \\
0.567\end{array}$ & 0.318 & $\begin{array}{r}1.25 \\
7\end{array}$ & -0.22 & 1.108 \\
\hline Count & 1201 & 1201 & 1201 & 1201 & 1201 & 1201 & 1201 & 1201 & 1201 & 1201 & 1201 & 1201 & 1201 \\
\hline
\end{tabular}

Source: developed by authors; the significance at $5 \%$.

\section{Correlation Matrix}

To get a better understanding, it is important to study the correlation among stocks in the market. In the table. 3 and table.4, information regarding the correlation among the considering companies have been discussed. The relationship may either be positive or negative. A positive correlation indicates that both the companies will move in the same direction and negative correlation shows the opposite scenario; i.e. the inverse relationship. The range of observed correlation coefficient is from .09 to .36 . Here, the highest positive correlation is observed between the stock 2 and stock 5 which is .36 , while the highest negative correlation is between stock 4 and stock 25 which is -.09 .

Table 3. Correlation among the Companies

\begin{tabular}{|c|c|c|c|c|c|c|c|c|c|c|c|c|c|}
\hline & $\begin{array}{c}\text { Stoc } \\
\mathbf{k} \\
1\end{array}$ & $\begin{array}{c}\text { Stoc } \\
\mathbf{k} \\
\mathbf{2}\end{array}$ & $\begin{array}{c}\text { Stoc } \\
\mathbf{k} \\
\mathbf{3}\end{array}$ & $\begin{array}{c}\text { Stoc } \\
\mathbf{k} \\
4\end{array}$ & $\begin{array}{c}\text { Stoc } \\
\mathbf{k} \\
\mathbf{5}\end{array}$ & $\begin{array}{c}\text { Stoc } \\
\mathbf{k} \\
\mathbf{6}\end{array}$ & $\begin{array}{c}\text { Stoc } \\
\mathbf{k} \\
7\end{array}$ & $\begin{array}{c}\text { Stoc } \\
\mathbf{k} \\
\mathbf{8}\end{array}$ & $\begin{array}{c}\text { Stoc } \\
\mathbf{k} \\
\mathbf{9}\end{array}$ & $\begin{array}{c}\text { Stoc } \\
\mathbf{k} \\
\mathbf{1 0}\end{array}$ & $\begin{array}{c}\text { Stoc } \\
\text { k } \\
11\end{array}$ & $\begin{array}{c}\text { Stoc } \\
\mathbf{k} \\
12\end{array}$ & $\begin{array}{c}\text { Stoc } \\
\mathbf{k} \\
\mathbf{1 3}\end{array}$ \\
\hline $\begin{array}{r}\text { Stoc } \\
\mathrm{k} 1\end{array}$ & 1 & & & & & & & & & & & & \\
\hline $\begin{array}{r}\text { Stoc } \\
\mathrm{k} 2 \\
\end{array}$ & 0.13 & 1 & & & & & & & & & & & \\
\hline $\begin{array}{r}\text { Stoc } \\
\mathrm{k} 3\end{array}$ & 0.06 & 0.07 & 1 & & & & & & & & & & \\
\hline $\begin{array}{r}\text { Stoc } \\
\mathrm{k} 4\end{array}$ & 0.2 & 0.09 & 0.21 & 1 & & & & & & & & & \\
\hline
\end{tabular}




\begin{tabular}{|c|c|c|c|c|c|c|c|c|c|c|c|c|c|}
\hline $\begin{array}{r}\text { Stoc } \\
\mathrm{k} 5\end{array}$ & 0.08 & 0.36 & 0 & 0.1 & 1 & & & & & & & & \\
\hline $\begin{array}{r}\text { Stoc } \\
\mathrm{k} 6\end{array}$ & 0.08 & 0.02 & 0.09 & 0.04 & 0.01 & 1 & & & & & & & \\
\hline $\begin{array}{r}\text { Stoc } \\
\mathrm{k} 7\end{array}$ & 0.08 & 0.11 & 0.05 & 0.1 & 0.1 & 0.28 & 1 & & & & & & \\
\hline $\begin{array}{r}\text { Stoc } \\
\mathrm{k} 8 \\
\end{array}$ & 0.11 & 0.13 & 0.06 & 0.12 & 0.11 & 0 & 0.09 & 1 & & & & & \\
\hline $\begin{array}{r}\text { Stoc } \\
\mathrm{k} 9 \\
\end{array}$ & 0.12 & 0.1 & 0.02 & 0.11 & 0.12 & 0.15 & 0.26 & 0.09 & 1 & & & & \\
\hline $\begin{array}{l}\text { Stoc } \\
\text { k 10 }\end{array}$ & 0.03 & $\begin{array}{r}- \\
0.01\end{array}$ & 0.02 & 0.01 & $\begin{array}{r}- \\
0.02\end{array}$ & 0.04 & 0 & 0.04 & $\begin{array}{r}- \\
0.01\end{array}$ & 1 & & & \\
\hline $\begin{array}{l}\text { Stoc } \\
\text { k } 11\end{array}$ & 0.03 & 0.03 & 0.05 & 0.01 & 0.04 & 0.02 & 0.04 & 0.03 & 0 & 0.01 & 1 & & \\
\hline $\begin{array}{l}\text { Stoc } \\
\text { k 12 }\end{array}$ & 0.04 & 0 & 0.02 & 0.03 & 0.03 & 0.04 & 0 & 0.02 & $\begin{array}{r}- \\
0.02 \\
\end{array}$ & 0 & 0.06 & 1 & \\
\hline $\begin{array}{l}\text { Stoc } \\
\text { k 13 }\end{array}$ & 0.1 & 0.01 & 0.06 & 0.06 & 0.03 & $\begin{array}{r}- \\
0.08\end{array}$ & 0.01 & 0.02 & 0.02 & 0 & $\begin{array}{r}- \\
0.02\end{array}$ & $\begin{array}{r}- \\
0.09\end{array}$ & 1 \\
\hline $\begin{array}{l}\text { Stoc } \\
\text { k 14 }\end{array}$ & 0.19 & 0.08 & 0.03 & $\begin{array}{r}- \\
0.01 \\
\end{array}$ & 0.06 & 0.07 & 0.12 & 0.09 & 0.15 & 0.01 & $\begin{array}{r}- \\
0.05\end{array}$ & 0 & 0.06 \\
\hline $\begin{array}{l}\text { Stoc } \\
\text { k 15 } \\
\end{array}$ & 0.03 & 0.08 & 0.01 & 0.08 & 0.02 & 0.05 & 0.11 & 0.03 & 0.33 & 0.01 & 0.01 & 0 & 0.01 \\
\hline $\begin{array}{l}\text { Stoc } \\
\text { k 16 }\end{array}$ & 0.05 & 0.15 & 0.06 & 0.07 & 0.18 & 0.05 & 0.05 & 0.1 & 0.07 & 0.03 & 0.01 & 0.06 & $\begin{array}{r}- \\
0.01\end{array}$ \\
\hline $\begin{array}{l}\text { Stoc } \\
\text { k 17 } \\
\end{array}$ & 0.08 & 0.2 & 0.11 & 0.12 & 0.15 & 0.03 & 0.09 & 0.12 & 0.1 & 0.02 & 0.01 & 0.01 & 0.01 \\
\hline $\begin{array}{l}\text { Stoc } \\
\text { k 18 }\end{array}$ & 0.22 & 0.19 & 0.11 & 0.24 & 0.12 & 0.06 & 0.18 & 0.14 & 0.18 & 0.03 & 0.02 & 0 & 0.06 \\
\hline $\begin{array}{l}\text { Stoc } \\
\text { k } 19 \\
\end{array}$ & 0.18 & 0.23 & 0.12 & 0.19 & 0.21 & 0.07 & 0.11 & 0.21 & 0.15 & 0.01 & 0 & $\begin{array}{r}- \\
0.01 \\
\end{array}$ & 0.06 \\
\hline $\begin{array}{l}\text { Stoc } \\
\mathrm{k} 20 \\
\end{array}$ & 0.17 & 0.17 & 0.12 & 0.24 & 0.15 & 0.01 & 0.11 & 0.06 & 0.14 & 0.02 & 0.02 & 0.03 & 0.06 \\
\hline $\begin{array}{l}\text { Stoc } \\
\mathrm{k} 21 \\
\end{array}$ & 0.01 & 0 & 0.01 & $\begin{array}{r}- \\
0.07 \\
\end{array}$ & 0 & 0 & $\begin{array}{r}- \\
0.07 \\
\end{array}$ & 0.01 & 0 & 0.02 & 0.02 & 0.02 & 0.01 \\
\hline $\begin{array}{l}\text { Stoc } \\
\mathrm{k} 22 \\
\end{array}$ & 0.18 & 0.13 & 0.03 & 0.05 & 0.16 & $\begin{array}{r}- \\
0.06 \\
\end{array}$ & 0.04 & 0.1 & 0.03 & 0.02 & $\begin{array}{r}- \\
0.02 \\
\end{array}$ & $\begin{array}{r}- \\
0.01 \\
\end{array}$ & 0.06 \\
\hline $\begin{array}{l}\text { Stoc } \\
\text { k 23 }\end{array}$ & 0.11 & 0.31 & 0.01 & 0.11 & 0.24 & $\begin{array}{r}- \\
0.02 \\
\end{array}$ & 0.03 & 0.11 & 0.06 & $\begin{array}{r}- \\
0.01\end{array}$ & 0.01 & 0.06 & 0.04 \\
\hline $\begin{array}{l}\text { Stoc } \\
\mathrm{k} 24 \\
\end{array}$ & 0.19 & 0.08 & 0.06 & 0.12 & 0.03 & 0.37 & 0.32 & 0.09 & 0.19 & $\begin{array}{r}- \\
0.01 \\
\end{array}$ & $\begin{array}{r}- \\
0.02 \\
\end{array}$ & 0 & 0.04 \\
\hline $\begin{array}{l}\text { Stoc } \\
\mathrm{k} 25 \\
\end{array}$ & 0.02 & 0 & 0.04 & $\begin{array}{r}- \\
0.09 \\
\end{array}$ & $\begin{array}{r}- \\
0.06 \\
\end{array}$ & 0 & 0.02 & 0.04 & 0.04 & $\begin{array}{r}- \\
0.03 \\
\end{array}$ & 0.03 & 0.01 & 0 \\
\hline $\begin{array}{l}\text { Stoc } \\
\mathrm{k} 26\end{array}$ & 0.01 & 0.01 & $\begin{array}{r}- \\
0.01\end{array}$ & 0.05 & 0.05 & 0.07 & 0.13 & 0.05 & 0.24 & 0.02 & 0.01 & 0.03 & 0.01 \\
\hline
\end{tabular}

Source: developed by authors

Table.4 Correlation among the Companies

\begin{tabular}{|c|c|c|c|c|c|c|c|c|c|c|c|c|c|}
\hline & $\begin{array}{c}\text { Stoc } \\
\mathbf{k} \\
14\end{array}$ & $\begin{array}{c}\text { Stoc } \\
\mathbf{k} \\
15\end{array}$ & $\begin{array}{c}\text { Stoc } \\
\mathbf{k} \\
16\end{array}$ & $\begin{array}{c}\text { Stoc } \\
\mathbf{k} \\
\mathbf{1 7}\end{array}$ & $\begin{array}{c}\text { Stoc } \\
\mathbf{k} \\
\mathbf{1 8}\end{array}$ & $\begin{array}{c}\text { Stoc } \\
\mathbf{k} \\
19\end{array}$ & $\begin{array}{c}\text { Stoc } \\
\mathbf{k} \\
\mathbf{2 0}\end{array}$ & $\begin{array}{c}\text { Stoc } \\
\mathbf{k} \\
\mathbf{2 1}\end{array}$ & $\begin{array}{c}\text { Stoc } \\
\mathbf{k} \\
\mathbf{2 2}\end{array}$ & $\begin{array}{c}\text { Stoc } \\
\mathbf{k} \\
\mathbf{2 3}\end{array}$ & $\begin{array}{c}\text { Stoc } \\
\mathbf{k} \\
\mathbf{2 4}\end{array}$ & $\begin{array}{c}\text { Stock } \\
25\end{array}$ & $\begin{array}{c}\text { Stoc } \\
\mathbf{k} \\
\mathbf{2 6}\end{array}$ \\
\hline $\begin{array}{l}\text { Stock } \\
14\end{array}$ & 1 & & & & & & & & & & & & \\
\hline $\begin{array}{l}\text { Stock } \\
15\end{array}$ & 0.1 & 1 & & & & & & & & & & & \\
\hline
\end{tabular}




\begin{tabular}{|c|c|c|c|c|c|c|c|c|c|c|c|c|c|}
\hline $\begin{array}{l}\text { Stock } \\
16\end{array}$ & $0.02^{-}$ & 0.02 & 1 & & & & & & & & & & \\
\hline $\begin{array}{l}\text { Stock } \\
17 \\
\end{array}$ & 0.05 & 0.02 & 0.16 & 1 & & & & & & & & & \\
\hline $\begin{array}{l}\text { Stock } \\
18 \\
\end{array}$ & 0.08 & 0.07 & 0.07 & 0.15 & 1 & & & & & & & & \\
\hline $\begin{array}{l}\text { Stock } \\
19\end{array}$ & 0.09 & 0.04 & 0.17 & 0.17 & 0.25 & 1 & & & & & & & \\
\hline $\begin{array}{l}\text { Stock } \\
20 \\
\end{array}$ & 0.11 & 0.06 & 0.09 & 0.17 & 0.27 & 0.22 & 1 & & & & & & \\
\hline $\begin{array}{l}\text { Stock } \\
21\end{array}$ & 0.04 & $\begin{array}{r}- \\
0.01\end{array}$ & 0.02 & 0.04 & $\begin{array}{r}-- \\
0.05\end{array}$ & 0.01 & 0.02 & 1 & & & & & \\
\hline $\begin{array}{l}\text { Stock } \\
22 \\
\end{array}$ & 0.04 & 0 & 0.08 & 0.16 & 0.12 & 0.15 & 0.13 & $\begin{array}{r}- \\
0.01\end{array}$ & 1 & & & & \\
\hline $\begin{array}{l}\text { Stock } \\
23 \\
\end{array}$ & 0 & 0.05 & 0.14 & 0.19 & 0.07 & 0.2 & 0.14 & 0.02 & 0.11 & 1 & & & \\
\hline $\begin{array}{l}\text { Stock } \\
24\end{array}$ & 0.16 & 0.05 & 0.03 & 0.06 & 0.1 & 0.13 & 0.08 & $\begin{array}{r}- \\
0.03\end{array}$ & 0.04 & 0.02 & 1 & & \\
\hline $\begin{array}{l}\text { Stock } \\
25\end{array}$ & 0.02 & $\begin{array}{r}- \\
0.01\end{array}$ & $\begin{array}{r}- \\
0.01 \\
\end{array}$ & 0 & 0 & 0.01 & $\begin{array}{r}- \\
0.02 \\
\end{array}$ & 0.09 & 0 & $\begin{array}{r}- \\
0.05 \\
\end{array}$ & 0.04 & 1 & \\
\hline $\begin{array}{l}\text { Stock } \\
26 \\
\end{array}$ & 0.07 & 0.25 & 0.03 & 0.03 & 0.05 & 0.04 & 0.02 & $\begin{array}{r}- \\
0.02\end{array}$ & 0 & 0.03 & 0.15 & -0.04 & 1 \\
\hline
\end{tabular}

Source: developed by authors

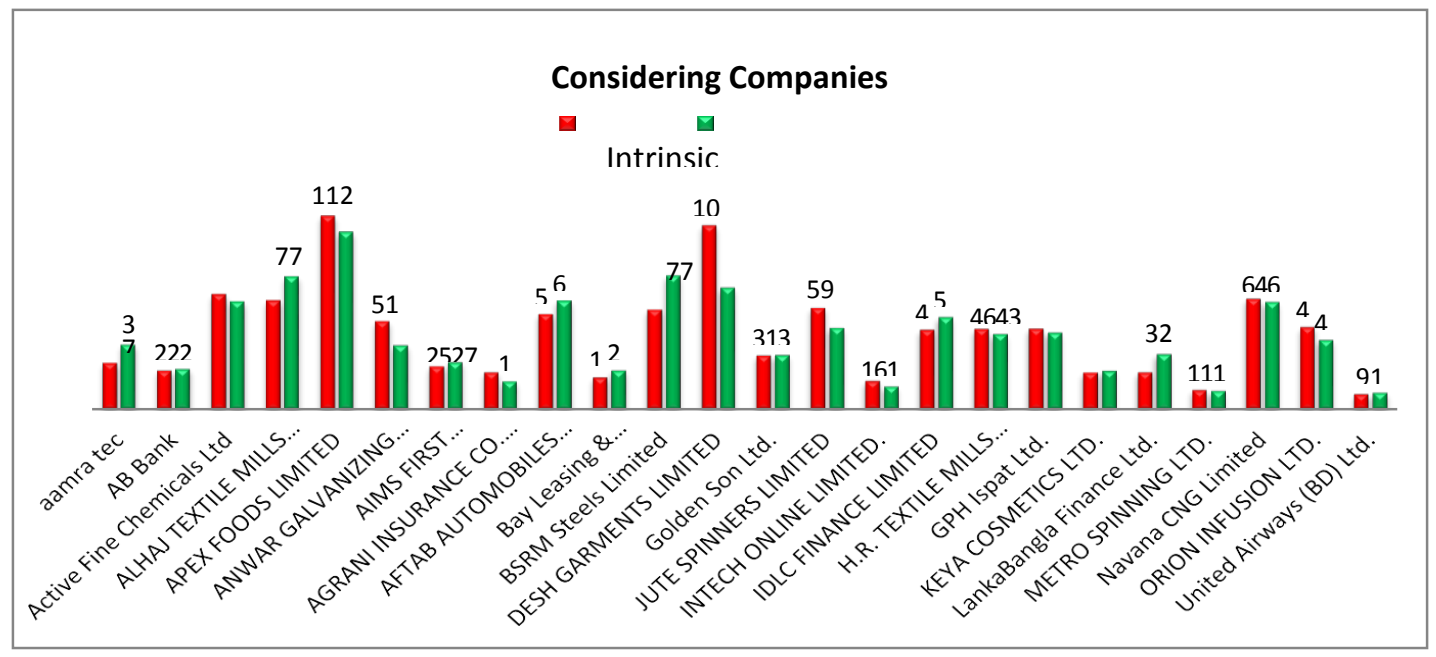

Graph - 1: Comparing Current Market Price and Intrinsic Value

Sources: Developed by authors

In the graph-1 above, the trading market price of companies along with their fair market values are disclosed. The red color columns represent the intrinsic values and the green color columns indicate the current market price. Graph -1 can be utilized to make an effective investment decision. One of the most notable undervalued stock is Desh Garments Ltd with an intrinsic price of Tk. 106 and the current market price is Tk. 70. This stock's price is very likely to increase in near future. On the other hand, BRSM Steel Ltd represents the highest overvalued stock with an intrinsic price of Tk. 65 and the current market price is Tk.77. This stock's price is expected to decline. 
After analyzing, we understand which companies are undervalued and which companies are overvalued. Then we take the decision to include the companies in our portfolio. If we look at the graph - 1, we can easily understand the position of the companies. Here, there are two colors in the bar chart, red and blue. The blue color indicates the intrinsic value and the red color indicates the market value. Sometimes the intrinsic values cross the current market value, at this point the company is undervalued. When the market values cross the intrinsic values these companies are overvalued. If we have any shares in my hand we should sell these in the market because the price will fall at any time.

Table-5: Analysing Overvalued or Undervalued

\begin{tabular}{|c|c|c|c|c|c|c|c|c|}
\hline $\begin{array}{c}\text { Company } \\
\text { Name }\end{array}$ & $\begin{array}{l}\text { Market } \\
\text { Return }\end{array}$ & Beta & $\begin{array}{c}\text { Risk } \\
\text { Premium } \\
\text { with } \\
\text { BETA }\end{array}$ & $\begin{array}{l}\text { The } \\
\text { required } \\
\text { rate of } \\
\text { return }\end{array}$ & $\begin{array}{l}\text { Current } \\
\text { Price* } \\
\text { Market } \\
\text { Return }\end{array}$ & $\begin{array}{c}\text { Intrinsic } \\
\text { Value }\end{array}$ & $\begin{array}{c}\text { Current } \\
\text { Market } \\
\text { Price }\end{array}$ & $\begin{array}{c}\text { Over } \\
\text { Valued } \\
\text { or } \\
\text { Under } \\
\text { Valued }\end{array}$ \\
\hline $\begin{array}{l}\text { Active Fine } \\
\text { Chemicals } \\
\text { Ltd }\end{array}$ & 0.14 & 0.86 & 0.06 & 0.13 & 8.6 & 66.69 & 62.2 & $\begin{array}{l}\text { Under } \\
\text { Valued }\end{array}$ \\
\hline $\begin{array}{l}\text { Apex Foods } \\
\text { Limited }\end{array}$ & 0.14 & 0.83 & 0.06 & 0.13 & 14.13 & 111.85 & 102.2 & $\begin{array}{l}\text { Under } \\
\text { Valued }\end{array}$ \\
\hline $\begin{array}{l}\text { Anwar } \\
\text { Galvanizing } \\
\text { Limited }\end{array}$ & 0.14 & 0.44 & 0.03 & 0.1 & 5.11 & 50.94 & 36.95 & $\begin{array}{l}\text { Under } \\
\text { Valued }\end{array}$ \\
\hline $\begin{array}{l}\text { Apex Foods } \\
\text { Limited }\end{array}$ & 0.14 & 0.83 & 0.06 & 0.13 & 14.13 & 111.85 & 102.2 & $\begin{array}{l}\text { Under } \\
\text { Valued }\end{array}$ \\
\hline $\begin{array}{l}\text { Anwar } \\
\text { Galvanizing } \\
\text { Limited }\end{array}$ & 0.14 & 0.44 & 0.03 & 0.1 & 5.11 & 50.94 & 36.95 & $\begin{array}{l}\text { Under } \\
\text { Valued }\end{array}$ \\
\hline $\begin{array}{l}\text { Agrani } \\
\text { Insurance } \\
\text { Co. Ltd. }\end{array}$ & 0.14 & 0.51 & 0.03 & 0.1 & 2.23 & 21.3 & 16.1 & $\begin{array}{l}\text { Under } \\
\text { Valued }\end{array}$ \\
\hline $\begin{array}{l}\text { Desh } \\
\text { Garments } \\
\text { Limited }\end{array}$ & 0.14 & 0.31 & 0.02 & 0.09 & 9.68 & 106.24 & 70 & $\begin{array}{l}\text { Under } \\
\text { Valued }\end{array}$ \\
\hline $\begin{array}{l}\text { Golden Son } \\
\text { Ltd. }\end{array}$ & 0.14 & 1 & 0.07 & 0.14 & 4.31 & 31.14 & 31.2 & $\begin{array}{l}\text { Under } \\
\text { Valued }\end{array}$ \\
\hline $\begin{array}{l}\text { Jute } \\
\text { Spinners } \\
\text { Limited } \\
\end{array}$ & 0.14 & 0.6 & 0.04 & 0.11 & 6.5 & 58.63 & 47 & $\begin{array}{l}\text { Under } \\
\text { Valued }\end{array}$ \\
\hline $\begin{array}{l}\text { Intech } \\
\text { Online }\end{array}$ & 0.14 & 0.61 & 0.04 & 0.11 & 1.82 & 16.29 & 13.15 & $\begin{array}{l}\text { Under } \\
\text { Valued }\end{array}$ \\
\hline
\end{tabular}


H.R. Textile

$0.14 \quad 0.86$

0.06

0.13

5.99

46.43

43.3 Under

Mills

Limited

\begin{tabular}{lccccccrl}
\hline $\begin{array}{l}\text { Gph Ispat } \\
\text { Ltd. }\end{array}$ & 0.14 & 0.9 & 0.06 & 0.13 & 6.12 & 46.55 & 44.3 & $\begin{array}{l}\text { Under } \\
\text { Valued }\end{array}$ \\
\hline $\begin{array}{l}\text { Lafarge } \\
\text { Surma }\end{array}$ & 0.14 & 0.22 & 0.02 & 0.09 & 16.25 & 190.79 & 117.55 & $\begin{array}{l}\text { Under } \\
\text { Valued }\end{array}$ \\
\hline
\end{tabular}

Cement Ltd.

\begin{tabular}{lllllllll}
\hline $\begin{array}{l}\text { Metro } \\
\text { Spinning }\end{array}$ & 0.14 & 0.93 & 0.06 & 0.13 & 1.47 & 10.99 & 10.6 & $\begin{array}{l}\text { Under } \\
\text { Valued }\end{array}$ \\
\hline
\end{tabular}

Ltd.

\begin{tabular}{lcccccccc}
\hline $\begin{array}{l}\text { Navana Cng } \\
\text { Limited }\end{array}$ & 0.14 & 0.93 & 0.06 & 0.13 & 8.54 & 63.93 & 61.8 & $\begin{array}{l}\text { Under } \\
\text { Valued }\end{array}$ \\
\hline $\begin{array}{l}\text { Orion } \\
\text { Infusion Ltd. }\end{array}$ & 0.14 & 0.67 & 0.05 & 0.12 & 5.52 & 47.54 & 39.9 & $\begin{array}{l}\text { Under } \\
\text { Valued }\end{array}$ \\
\hline $\begin{array}{l}\text { Aamra } \\
\text { technologies } \\
\text { limited }\end{array}$ & 0.138 & 1.789 & 0.122 & 0.192 & 5.142 & 26.774 & 37.2 & Over \\
\hline AB Bank & 0.138 & 1.08 & 0.074 & 0.144 & 3.207 & 22.316 & 23.2 & $\begin{array}{l}\text { Over } \\
\text { Valued }\end{array}$ \\
\hline $\begin{array}{l}\text { Aci } \\
\text { Formulations }\end{array}$ & 0.138 & 1.413 & 0.096 & 0.166 & 32.552 & 195.659 & 235.5 & $\begin{array}{l}\text { Over } \\
\text { Valued }\end{array}$ \\
Limited & & & & & & & & \\
Valued
\end{tabular}

Limited

\begin{tabular}{|c|c|c|c|c|c|c|c|c|}
\hline $\begin{array}{l}\text { Alhaj Textile } \\
\text { Mills } \\
\text { Limited }\end{array}$ & 0.138 & 1.435 & 0.098 & 0.168 & 10.588 & 63.051 & 76.6 & $\begin{array}{l}\text { Over } \\
\text { Valued }\end{array}$ \\
\hline $\begin{array}{l}\text { Aims First } \\
\text { Guaranteed } \\
\text { Mutual } \\
\text { Fund. }\end{array}$ & 0.138 & 1.2 & 0.082 & 0.152 & 3.76 & 24.758 & 27.2 & $\begin{array}{l}\text { Over } \\
\text { Valued }\end{array}$ \\
\hline
\end{tabular}

\begin{tabular}{lllllllll}
\hline $\begin{array}{l}\text { Aftab } \\
\text { Automobiles }\end{array}$ & 0.138 & 1.282 & 0.087 & 0.157 & 8.66 & 54.995 & 62.65 & Over \\
Valued
\end{tabular}

Limited

\begin{tabular}{|c|c|c|c|c|c|c|c|c|}
\hline $\begin{array}{l}\text { Bangas } \\
\text { Limited }\end{array}$ & 0.138 & 1.101 & 0.075 & 0.145 & 45.974 & 316.821 & 332.6 & $\begin{array}{l}\text { Over } \\
\text { Valued }\end{array}$ \\
\hline $\begin{array}{l}\text { Bay Lessing } \\
\text { and } \\
\text { investment } \\
\text { ltd. }\end{array}$ & 0.138 & 1.43 & 0.098 & 0.168 & 3.096 & 18.476 & 22.4 & $\begin{array}{l}\text { Over } \\
\text { Valued }\end{array}$ \\
\hline $\begin{array}{l}\text { Bsrm Steels } \\
\text { Limited }\end{array}$ & 0.138 & 1.683 & 0.115 & 0.185 & 10.65 & 57.62 & 77.05 & $\begin{array}{l}\text { Over } \\
\text { Valued }\end{array}$ \\
\hline $\begin{array}{l}\text { Idlc Finance } \\
\text { Limited }\end{array}$ & 0.138 & 1.316 & 0.09 & 0.16 & 7.34 & 45.931 & 53.1 & $\begin{array}{l}\text { Over } \\
\text { Valued }\end{array}$ \\
\hline
\end{tabular}




\begin{tabular}{lcccccccc}
$\begin{array}{l}\text { Keya } \\
\text { Cosmetics } \\
\text { Ltd. }\end{array}$ & 0.138 & 1.103 & 0.075 & 0.145 & 3.069 & 21.124 & 22.2 & $\begin{array}{l}\text { Over } \\
\text { Valued }\end{array}$ \\
\hline $\begin{array}{l}\text { Lankabangla } \\
\text { Finance Ltd. }\end{array}$ & 0.138 & 2.001 & 0.137 & 0.207 & 4.396 & 21.281 & 31.8 & $\begin{array}{l}\text { Over } \\
\text { Valued }\end{array}$ \\
\hline $\begin{array}{l}\text { Libra } \\
\text { Infusions }\end{array}$ & 0.138 & 1.262 & 0.086 & 0.156 & 50.978 & 326.553 & 368.8 & Over \\
\hline $\begin{array}{l}\text { Mjl } \\
\text { Bangladesh } \\
\text { Limited }\end{array}$ & 0.138 & 0.618 & 0.042 & 0.112 & 16.007 & 142.736 & 115.8 & Over \\
\hline $\begin{array}{l}\text { United } \\
\text { Airways } \\
\text { (Bd) Ltd. }\end{array}$ & 0.138 & 1.201 & 0.082 & 0.152 & 1.313 & 8.643 & 9.5 & Over \\
\hline
\end{tabular}

Source: developed by authors

The table -5 discloses the execution of our methodology that has been discussed earlier. It shows all the considering companies market information in regards to market return, beta, risk premium with beta, required rate of return, intrinsic price, current market price and lastly, the decision whether the stock is overvalued or undervalued. It can be observed that, there is not a single company whose current market price is equal to their calculated intrinsic value. It suggests that the market is not weak form efficient. Indeed, it is an inefficient market according to the findings. If an investor invests in undervalued stocks, the price is expected to increase in near future and there is a possibility to gain an abnormal profit. On the contrary, investors should sell their overvalued stocks before they starts losing their prices.

\section{CONCLUSION}

The study examines stock valuation of various corporations in the context of weak form market efficiency on Bangladesh capital market. Since the stock market in Bangladesh is considered inefficient, the investors can make an arbitrage profit by analyzing the market. In this study, the Capital Asset Pricing Model (CAPM) was used to find out the intrinsic value of stocks. The outcome of this empirical study shows that not a single company among the considering companies are traded at fair price. It is either overvalued or undervalued. It proves that the market is not efficient. In this study, five out of twenty-six stocks in the market were found overvalued and it is suggested that those stocks should be sold as their price may fall in near future. Therefore, technical analysts can earn abnormal profit due to market inefficiency by using CAPM. This study may be used for conducting further research, for example, stock valuation from a dynamic economic perspective, stock valuation in the context of various stock markets of different countries, and achieving arbitrage profit by using CAPM. 


\section{REFERENCE}

Ahmed, R., \& Hossain, M. D. (2018). Testing Weak form Market Efficiency: Empirical Evidence from Selected Asian Stock Markets. Asian Economic and Financial Review, 8(6), 790-798.

Bakshi, G., \& Chen, Z. (2005). Stock valuation in dynamic economies. Journal of Financial Markets, 8(2), 111-151.

Carpenter, J. N. (1998). The exercise and valuation of executive stock options1. Journal of Financial Economics, 48(2), 127-158.

Do, H. Q., Bhatti, M. I., \& Konya, L. (2016). On ASEAN Capital Market and Industry Integration: A Review. Corporate Ownership and Control, 2(1), 823.

French, C. W. (2003). The Treynor capital asset pricing model. Journal of Investment Management, 1(2), 60-72.

Gupta, R. and P. K. Basu (2007). Weak form efficiency in Indian stock markets', international business \& economics research journal, 6 (3), 57-64.

Jennergren, L. P., \& Näslund, B. (1993). A Comment on" Valuation of Executive Stock Options and the FASB Proposal". The Accounting Review, 68(1), 179-183.

Jagannathan, R., \& Wang, Z. (1996). The conditional CAPM and the cross-section of expected returns. The Journal of finance, 51(1), 3-53.

Kumar, H., \& Jawa, R. (2017). Efficient Market Hypothesis and Calendar Effects: Empirical Evidences from the Indian Stock Markets.

Lewellen, J., \& Nagel, S. (2006). The conditional CAPM does not explain assetpricing anomalies. Journal of financial economics, 82(2), 289-314.

Malkiel, B. G., \& Fama, E. F. (1970). Efficient capital markets: A review of theory and empirical work. The journal of Finance, 25(2), 383-417.

Mossin, J. (1966). Equilibrium in a capital asset market. Econometrica: Journal of the econometric society, 768-783.

Mobarek, A., Mollah, A. S., \& Bhuyan, R. (2008). Market efficiency in emerging stock market: Evidence from Bangladesh. Journal of Emerging Market Finance, 7(1), 17-41.

Oprean, C., \& Tanasescu, C. (2014). Effects of behavioural finance on emerging capital markets. Procedia Economics and Finance, 15, 1710-1716.

Pástor, L., \& Pietro, V. (2003). Stock valuation and learning about profitability. The Journal of Finance, 58(5), 1749-1789.

Poshakwale, S. (1996). Evidence on weak form efficiency and day of the week effect in the Indian stock market. Finance India, 10(3), 605-616.

Pitkethly, R. (1997). The valuation of patents: a review of patent valuation methods with consideration of option based methods and the potential for further research. Research Papers in Management Studies-University of Cambridge Judge Institute of Management Studies.

Rahman, M. S., Simon, H. M., \& Hossain, M. M. (2016). An Empirical Analysis of Weak Form Market Efficiency: Evidence from Chittagong Stock Exchange (CSE) of Bangladesh.

Sunder, S. (1975). Stock price and risk related to accounting changes in inventory valuation. The Accounting Review, 50(2), 305-315. 
Shahid, M. N., \& Sattar, A. (2017). Behavior of Calendar Anomalies, Market Conditions and Adaptive Market Hypothesis: Evidence from Pakistan Stock Exchange. Pakistan Journal of Commerce \& Social Sciences, 11(2).

Sharpe, W. F. (1964). Capital asset prices: A theory of market equilibrium under conditions of risk. The journal of finance, 19(3), 425-442.

Tuyon, J., \& Ahmad, Z. (2016). Behavioural finance perspectives on Malaysian stock market efficiency. Borsa Istanbul Review, 16(1), 43-61.

Treynor, J. L. (1961). Market value, time, and risk.

Woo, C. Y. (1984). An empirical test of value-based planning models and implications. Management Science, 30(9), 1031-1050. 